\title{
A Retrospective Quality Study of Hemodialysis Catheter-Related Bacteremia in a Danish Hospital
}

\author{
Sophie Kaarup1, Bente Olesen², Mahshid Pourarsalan1, Lene Boesby¹, Lisbet Brandi1 \\ ${ }^{1}$ Department of Cardiology, Nephrology and Endocrinology, Nordsjaellands Hospital, University of Copenhagen, Copenhagen, Denmark \\ ${ }^{2}$ Department of Clinical Microbiology, Herlev and Gentofte Hospital, University of Copenhagen, Copenhagen, Denmark \\ Email: sophie.kaarup@hotmail.com
}

How to cite this paper: Kaarup, S., Olesen, B., Pourarsalan, M., Boesby, L. and Brandi, L. (2016) A Retrospective Quality Study of Hemodialysis Catheter-Related Bacteremia in a Danish Hospital. Open Journal of Nephrology, 6, 111-121.

http://dx.doi.org/10.4236/ojneph.2016.64014

Received: September 16, 2016

Accepted: November 8, 2016

Published: November 11, 2016

Copyright (c) 2016 by authors and Scientific Research Publishing Inc. This work is licensed under the Creative Commons Attribution International License (CC BY 4.0).

http://creativecommons.org/licenses/by/4.0/

(c) (i) Open Access

\begin{abstract}
Background: Hemodialysis catheter-related bacteremia (HD CRB) is a major complication of long-term hemodialysis (HD) therapy and bacteremia is secondary only to cardiovascular disease as the leading cause of death in patients receiving renal replacement therapy. A large part may be preventable and surveillance is a critical aspect of infection control and prevention. Aim: To analyze incidence, causative species, and treatment of HD CRB in adult chronic HD patients at Nordsjaellands Hospital (NOH), Denmark. Methods: All episodes of bacteremia in the Department of Cardiology, Nephrology and Endocrinology (KNEA), NOH from 2010 to 2013 were analyzed. Inclusion criteria: Adult chronic HD patients with a tunneled dialysis catheter diagnosed with HD CRB. Causative microorganism and antimicrobial treatment were recorded for each episode. Findings: Ninety-nine episodes of HD CRB in 72 patients were found with a mean incidence rate of $0.9 / 1000$ catheter-days. Grampositive bacteria were isolated in $71 \%$ of the episodes, gram-negative bacteria in $25 \%$, both in 3\%, and yeast in 1\%. The most frequently isolated microorganisms were Staphylococcus aureus (33\%), Coagulase-negative staphylococci (29\%), enterobacteriaceae $(20 \%)$ and enterococci $(8 \%)$. The most commonly used empiric antimicrobials were cefuroxime and vancomycin and the overall efficacy was $77 \%$. Conclusion: The well-functioning infection prevention strategy seems to be successful resulting in a relatively low incidence rate of HD CRB compared with that shown in international studies. The high proportion of gram-negative bacteria raises the question as to whether future antimicrobial guidelines should cover both gram-positive and gramnegative bacteria.
\end{abstract}

\section{Keywords}

Hemodialysis, Bacteremia, Microbiology, Surveillance, Quality Improvement 


\section{Introduction}

Bacteremia is a serious condition associated with substantial morbidity and a high shortterm mortality rate [1] [2] [3] [4]. In Denmark, about 35\% of the bacteremic episodes are nosocomial and approximately $20 \%$ are health-care acquired (patients with a hospital stay in the 30 days before hospital admission or who have regular hospital visits (e.g. for hemodialysis (HD) or chemotherapy)) [3]. This means that a large part of this serious problem may be preventable; consequently, focus on establishing an effective infection prevention strategy is paramount.

Patients with end-stage renal disease receiving chronic $\mathrm{HD}$ through a central venous catheter (CVC) are more susceptible to bacteremia than others, due to the direct access to the bloodstream, several comorbidities and an impaired immune system.

In 2013 approximately 2000 patients received chronic HD treatment in Denmark [5]. International studies have shown that the incidence rate of HD catheter-related bloodstream infections (CRBSI) is between 0.45 and 5.5 episodes per 1000 catheter-days and the hazard ratio for bacteremia in patients with tunneled CVCs compared with patients with native arterio-venous (AV) fistulas is approximately 5 [6]-[11].

Interventions proven to be effective in reducing the incidence rate of catheter-related bacteremia in HD patients are: surveillance, good hand hygiene, hygiene audits, aseptic techniques, staff and patient education, and reduction in the number of HD catheters [12] [13] [14].

Nevertheless, it is estimated that almost $30 \%$ of HD patients depend on CVCs as their primary vascular access and for some of these patients the use of HD catheters is unavoidable [13] [15].

The aim of this study was to analyze incidence, causative species, and treatment of hemodialysis catheter-related bacteremia (HD CRB) in adult chronic HD patients at Nordsjaellands Hospital (NOH), Denmark from 2010-2013.

\section{Methods}

\subsection{Study Design}

A single center, retrospective, quality study performed on adult chronic HD patients diagnosed with HD CRB in Department of Cardiology, Nephrology and Endocrinology (KNEA), NOH from 2010 to 2013.

Approximately 150 patients at $\mathrm{NOH}$ are on chronic $\mathrm{HD}$ treatment and approximately $40 \%$ have a tunneled CVC [5].

\subsection{Study Population}

All cases of bacteremia in KNEA, NOH from 2010-2013 were screened by reviewing the unitary electronic health records (EHR). Eligible patients were aged $\geq 18$ years, on chronic HD through a tunneled CVC and diagnosed with HD CRB in the study period. Patients receiving HD trough another type of vascular access or with other sources of infection were excluded. 


\subsection{Data Collection}

The EHR of the identified patients were reviewed by a nephrologist at $\mathrm{NOH}$ and clinical and demographic variables were recorded in a database. The following variables were collected: civil registration number [16], age, name, sex, vascular access type (HD catheter/AV fistula/graft), catheter type (permanent/acute), date of positive blood sample collection, location of blood sample collection, and type of microorganism isolated.

Department of Clinical Microbiology, Herlev and Gentofte Hospital, University of Copenhagen, routinely identified the microorganisms cultured from blood and performed antibiotic susceptibility testing. The antibiograms were retrospectively retrieved and analyzed in December 2014.

Information on the antimicrobial treatment was retrieved from the EHR, which include an electronic medication module. Antimicrobial treatment was analyzed in relation to the antibiograms of the microorganisms isolated from blood cultures.

\subsection{Study Definitions}

A chronic HD patient was defined as a patient on HD through a tunneled permanent CVC. A blood culture typically includes four vials taken from the catheter and/or four vials taken from a peripheral vein (two aerobe and two anaerobe, respectively).

An episode of HD CRB was defined as a positive blood culture with a bacterial or fungal isolate taken from the CVC or a peripheral vein of a patient on chronic HD therapy with no other identified source of infection. An episode was considered polymicrobial if more than one microorganism was isolated from the blood on the same date. Two episodes of HD CRB in the same patient were defined as two separate positive blood cultures with different microorganisms taken at intervals of more than 24 hours or two positive blood cultures with the same isolated microorganism taken at an interval of more than 28 days.

The empiric antimicrobials were defined as all antimicrobials given before knowing the species of the isolated microorganisms. The empiric treatment was considered effective if all bacteria/fungi cultured from the blood were susceptible to one or more of the given empiric antimicrobials.

All episodes were considered as health-care associated infections (HCAI) according to the definition from Friedman et al. and the European Centre for Disease Prevention and Control (ECDC) [17] [18].

\subsection{Outcome}

The outcomes of the study were the incidence rate of HD CRB per 1000 catheter-days, the frequencies of the different bacterial/fungal isolates and the efficacy of the empiric antimicrobials.

\subsection{Statistical Analysis}

The incidence rate of HD CRB per 1000 catheter-days was calculated for each quarter in the four-year study period. 
Catheter-days is an estimate found by counting the number of tunneled catheters in use per quarter and multiplying it by the number of days in the quarter.

Data are presented as median, range and interquartile range (IQR). Frequency distribution is presented as histograms and a pie diagram. Fisher's exact test was used for comparison of microbial isolates in men and women, $\mathrm{p}<0.05$ was considered significant.

\subsection{Ethics}

The study was approved by the Regional Scientific Ethics Committee (H-2-2014-FSP-73).

\section{Results}

\subsection{Patient Characteristics}

We identified 72 patients with HD CRB; 48 (67\%) were male and the patients had a median age of 66 years (IQR 52 - 71 years). Of those identified, 55 (76\%) had one episode of HD CRB in the study period, 10 (14\%) had two episodes, five (7\%) had three episodes and two (3\%) had more than three episodes (one patient had four and one patient had five episodes).

\subsection{Incidence Rates}

The mean incidence rate of HD CRB was 0.9 episodes/1000 catheter-days (range: 0.1 1.6). The incidence rate was stable during the study period with the lowest incidence rate found in the fourth quarter of $2011(0.1)$ and the highest incidence rate found in the first and third quarter of 2013 (1.6) (Figure 1, Table 1).

\subsection{Microbiology and Antibiograms}

During the study period, 99 episodes of HD CRB were found of which five (5\%) were

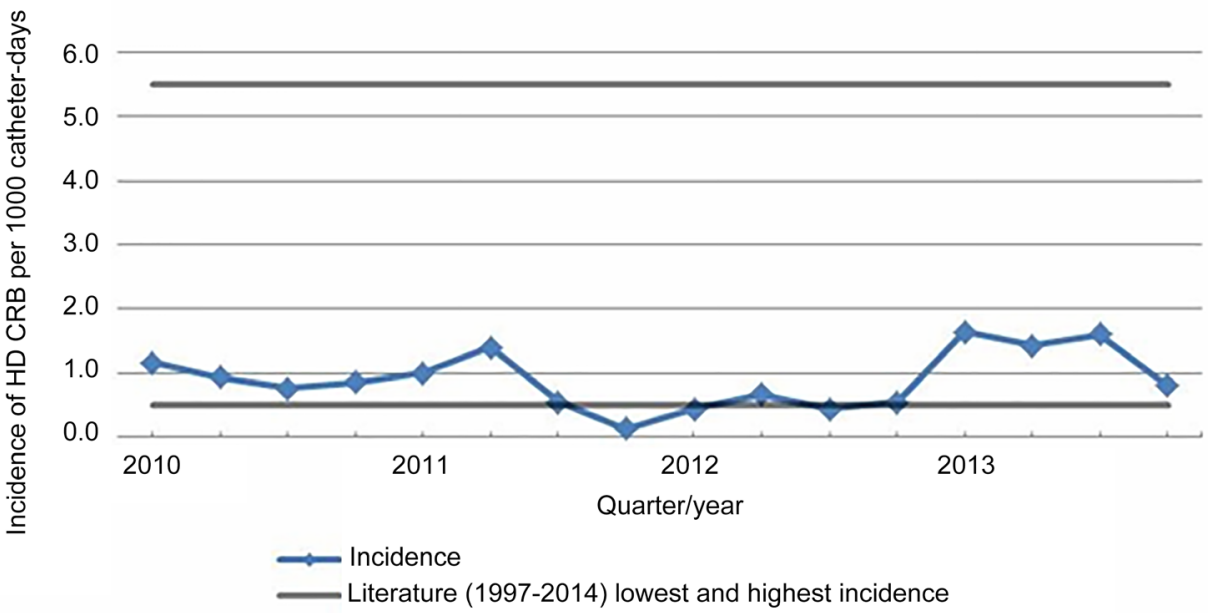

Figure 1. Incidence rate of HD CRB in adult chronic HD patients, at Nordsjaellands Hospital compared with international studies [6] [7] [8] [9] The incidence rate of HD CRB episodes is shown per 1000 catheter-days for every quarter in the surveillance period (2010-2013). 
Table 1. The calculated incidence (the number of HD CRB episodes per 1000 catheter-days) per quarter and per year in the study period.

\begin{tabular}{|c|c|c|c|c|}
\hline Year & Quarter & Episodes & Catheter-days & Incidence \\
\hline \multicolumn{5}{|l|}{2010} \\
\hline & 1 & 10 & 8550 & 1.2 \\
\hline & 2 & 8 & 8554 & 0.9 \\
\hline & 3 & 6 & 7912 & 0.8 \\
\hline & 4 & 7 & 8188 & 0.9 \\
\hline & & 31 & 33,204 & 0.9 \\
\hline \multicolumn{5}{|l|}{2011} \\
\hline & 1 & 7 & 7020 & 1.0 \\
\hline & 2 & 10 & 7150 & 1.4 \\
\hline & 3 & 4 & 7380 & 0.5 \\
\hline & 4 & 1 & 7380 & 0.1 \\
\hline & & 22 & 28,930 & 0.8 \\
\hline \multicolumn{5}{|l|}{2012} \\
\hline & 1 & 4 & 8918 & 0.4 \\
\hline & 2 & 6 & 8918 & 0.7 \\
\hline & 3 & 4 & 9016 & 0.4 \\
\hline & 4 & 5 & 9016 & 0.6 \\
\hline & & 19 & 35,868 & 0.5 \\
\hline \multicolumn{5}{|l|}{2013} \\
\hline & 1 & 8 & 4860 & 1.6 \\
\hline & 2 & 7 & 4914 & 1.4 \\
\hline & 3 & 8 & 4968 & 1.6 \\
\hline & 4 & 4 & 4968 & 0.8 \\
\hline & & 27 & 19,710 & 1.4 \\
\hline
\end{tabular}

polymicrobial. We found 104 bacterial/fungal isolates (103 bacterial, 1 fungal). Among the 99 episodes of HD CRB, 70 (71\%) were caused by gram-positive bacteria, 25 (25\%) were caused by gram-negative bacteria, three $(3 \%)$ were caused by both gram-positive and gram-negative bacteria and one episode (1\%) was caused by yeast (Candida tropicalis).

The most frequently isolated microorganisms were Staphylococcus aureus (33\%), Coagulase-negative staphylococci (CoNS; 29\%), and Enterobacter cloacae (8\%) (Figure 2).

No significant difference in microbial isolates between men and women was observed (Data not shown).

Antibiograms for 92 (93\%) episodes could be retrieved. No methicillin-resistant Staphylococcus aureus (MRSA), extended spectrum beta-lactamase (ESBL)-producing bacteria, Carbapenemase producing organisms (CPO) or vancomycin-resistant enterococcus (VRE) were found. 


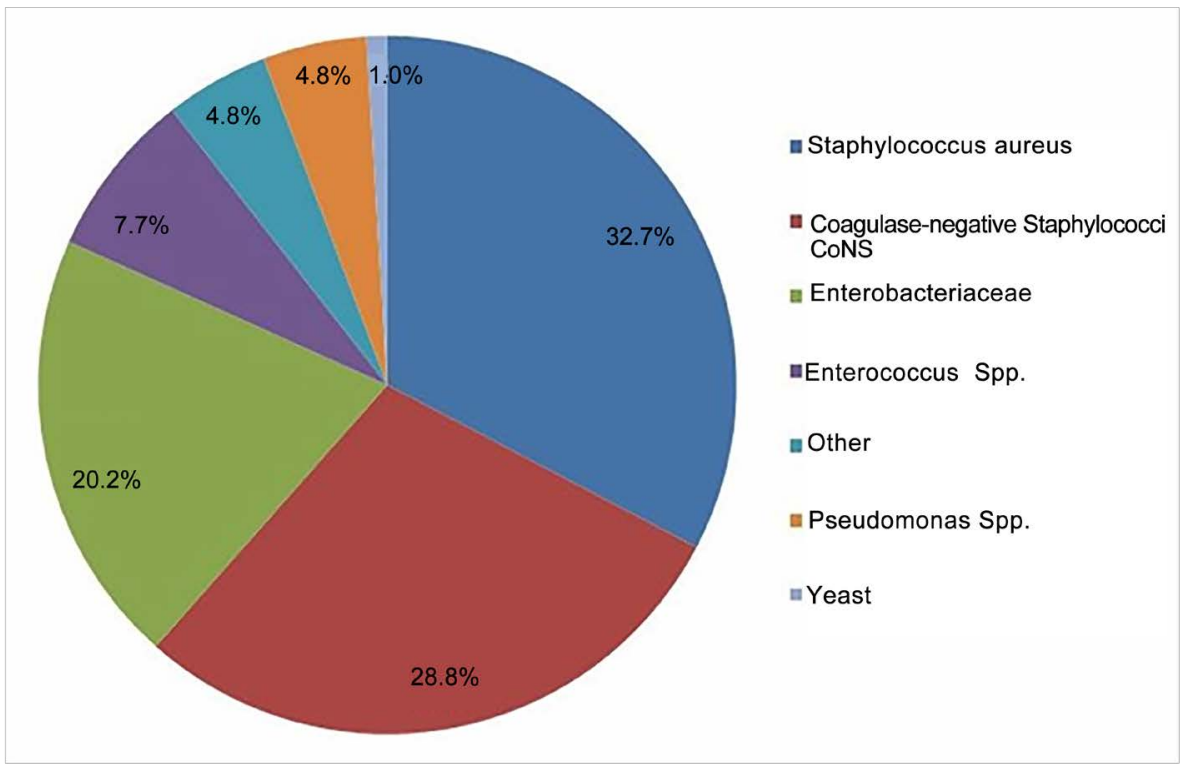

Figure 2. Distribution (\%) of microorganisms responsible for episodes of HD CRB in adult chronic HD patients, at Nordsjaellands Hospital from 2010-2013. A total of 99 episodes of HD CRB occurred in the four-year study period with 104 isolated microorganisms. Coagulase-negative staphylococci; CoNS: Staphylococcus epidermidis (17.3\%), unspecified CoNS (8.7\%), Staphylococcus hominis (1\%), Staphylococcus capitis (1\%), and Staphylococcus lugdunensis (1\%). Enterobacteriaceae: Enterobacter cloacae (7.7\%), Escherichia coli (5.8\%), Aeromonas caviae (1.9\%), Klebsiella pneumoniae (1\%), Klebsiella oxytoca (1\%), and Citrobacter koseri (1\%). Enterococcus Spp.: Enterococcus faecalis (4.8\%), and Enterococcus faecium (2.9\%). Other: Streptococcus mitis (1.9\%), Agrobacterium species (1\%), Bacteroides fragilis (1\%), and Micrococcus luteus (1\%). Pseudomonas Spp: Pseudomonas aeruginosa (2.9\%), and Stenotrophomonas maltophilia (1.9\%). Yeast: Candida tropicalis (1\%).

\subsection{Empiric Antimicrobials}

Empiric antimicrobials were given in 77 episodes (78\%) and the overall efficacy was $77 \%$. Fourteen types of empiric antimicrobials from seven different classes (penicillins, cephalosporins, macrolides, glycopeptides, quinolones, carbapenems, and others (fluconazole and metronidazole)) were used in the study period; the most frequently used were cefuroxime (35 episodes) and vancomycin (27 episodes) (Table 2).

Two or more types of empiric antimicrobials were administered in $44(44 \%)$ episodes.

\section{Discussion}

In the present retrospective quality study of bacteremia in chronic HD patients, the mean incidence rate of $\mathrm{HD} \mathrm{CRB}$ at $\mathrm{NOH}$ was 0.9 episodes/1000 catheter-days, which is low compared with that found in international studies [6] [7] [8] [9] [19]. Due to differences in methods and definitions, several international studies are not directly comparable with the present study. Some studies have investigated the incidence of HD $\mathrm{CRB}$ in dialysis patients with all types of CVCs, e.g. the acute single lumen catheters 
Table 2. List of the types of empiric antimicrobials used and the number of episodes they were used in.

\begin{tabular}{rc}
\hline Empiric Antimicrobials (Type) & Count \\
\hline Cefuroxime & 35 \\
Vancomycin & 27 \\
Ciprofloxacin & 18 \\
Dicloxacillin & 14 \\
Meropenem & 8 \\
Metronidazole & 6 \\
Penicillin & 6 \\
Gentamycin & 5 \\
Amoxacillin/Clavulanate & 3 \\
Flucloxacillin & 2 \\
Piperacillin/Tazobactam & 2 \\
Cephalexin & 1 \\
Fluconazole & 1 \\
Roxithromycin & 1 \\
\hline
\end{tabular}

[19], while others have defined an HD CRB differently due to differences in national guidelines, different equipment or different means of data collection. Furthermore, many studies calculate the incidence in episodes per person-days or years, which cannot be directly converted to the catheter-days, as used in the present study.

One study, from 2014 in North America by Rosenblum et al. [19], reported a bloodstream infection (BSI) rate of $0.81 / 1000$ catheter-days in a catheter hygiene intervention group and a BSI rate of 1.04/1000 catheter-days in their matched control group. Rosenblum et al. defined two separate BSIs as two positive blood cultures with 21 days between blood draws, whereas the present study defined it as two positive blood cultures with 28 days between the draws. In another study from North America in 2014 by Moore et al. [6], the incidence of CRBSI in all dialysis patients with a tunneled cuffed catheter was reported to be $0.45 / 1000$ catheter-days for a patient group treated with an antibiotic lock solution versus $1.68 / 1000$ catheter-days for the same group treated with the standard heparin lock. The study defined a CRBSI according to criteria established by the Centers for Disease Control and Prevention (CDC), which is in accordance with the definition used in the present study.

The incidence rate in the present study was continuously low during the four-year study period, but reached a maximum in the first and third quarter of 2013 (Figure 1, Table 1). This small increase in incidence rate could be explained by a remarkably low number of catheter-days in 2013 compared with the other three years in the study period (Table 1). The low number could be due to a change in the hospital's geographical up-take area in 2012, which reduced the number of HD patients at NOH.

The most frequently isolated microorganisms in both men and women were Staphy- 
lococcus aureus and CoNS, which is in accordance with other studies [6] [9] [20] [21].

An unexpected finding was the relatively high prevalence of bacteremic episodes caused by gram-negative bacteria (27\%). However, the results of this surveillance program largely follow those of recent studies [4] [8] [11] [20]. Alexandraki et al. investigated the five-year pattern of microorganisms causing bacteraemias in HD patients with an HD catheter and found a significant increase in the incidence of single gramnegative organisms and polymicrobial bacteremia [21]. This trend might be due to an increasing degree of immunodeficiency in the dialysis patients or a failed empiric antibiotic policy, which focuses only on treating the expected gram-positive microorganisms.

During the surveillance period, the most frequently used empiric antimicrobials were cefuroxime and vancomycin, with an overall empiric treatment efficacy of $77 \%$. Notably, cefuroxime was recommended as the empiric antibiotic treatment of sepsis without known focus at $\mathrm{NOH}$ in the study period. The recommendation was changed in 2014.

In a recent Danish study of bacteremia by Boel et al. [22], an efficacy of empirical antimicrobial treatment of minimum $90 \%$ was suggested as acceptable; however, this study differed significantly from the present study as it comprised mostly communityacquired infections and only few patients with an HD catheter. Accordingly, we consider an efficacy of empiric antimicrobials of $77 \%$ as acceptable.

In contrast to several other studies, we did not find any MRSA, ESBL-producing bacteria, CPO or VRE [6] [9] [20] [21] [23]. In general, Denmark has a low level of antibiotic resistance compared with many other countries [24].

A well-organized prevention program including surveillance as well as engaging patients and staff in preventive strategies has been reported to have a pronounced beneficial effect on the incidence of infections in dialysis units [25] [26] [27] [28] [29]. The relatively low average incidence rate of HD CRB found in this study is probably a result of the extensive prevention bundle approach found at $\mathrm{NOH}$, consisting of a surveillance program, a vascular access group where dialysis nurses and nephrologists have a special focus on establishing the optimal vascular access for every HD patient, hand hygiene education and audits for both staff and patients and a special no-touch method developed in the daily work with the dialysis patients where no hands or gloves are in direct contact with the head of the dialysis catheter when connecting and disconnecting to the dialysis machine.

Compared with the recommendations from the CDC [14], the infection prevention strategy at $\mathrm{NOH}$ follows eight of the nine recommendations.

The ninth recommendation regarding application of antimicrobial ointment to the catheter exit-site during dressing changes is not a part of the local guidelines.

This study is retrospective and observational in nature and therefore has its limitations. 1) Due to the observational nature of the study, there is a risk of selection bias. 2) It is not always possible to definitively determine the source of a bacteremia from electronic patient records when a clinical examination of the patient cannot be performed. 3) There may have been patients with HD CRB hospitalized outside the Department of 
Cardiology, Nephrology and Endocrinology, $\mathrm{NOH}$ and thereby not included in this study, but it is considered to be a very few patients, if any.

The strength of this study is that monitoring of CRB using the benefits of the Danish Civil Registration System has been routine for several years in the department, which makes the data, albeit retrospective, very robust.

\section{Conclusion}

In conclusion, the $\mathrm{NOH}$ infection prevention strategy seems to be successful resulting in a relatively low incidence rate of hemodialysis catheter-related bacteremia during the study period 2010-2013. The unexpected high proportion of gram-negative bacteria, however, raises the question as to whether future antimicrobial guidelines ought to cover both gram-positive and gram-negative bacteria.

\section{Acknowledgements}

We thank Niels Frimodt-Møller for reading the manuscript and Lisbeth B. Nielsen for retrieving data.

\section{Funding Source}

The research was supported by a research grant from $\mathrm{NOH}$.

\section{References}

[1] Lewis, S.S. and Sexton, D.J. (2013) Metastatic Complications of Bloodstream Infections in Hemodialysis Patients. Seminars in Dialysis, 26, 47-53. http://dx.doi.org/10.1111/sdi.12031

[2] Goto, M. and Al-Hasan, M.N. (2013) Overall Burden of Bloodstream Infection and Nosocomial Bloodstream Infection in North America and Europe. Clinical Microbiology and Infection, 19, 501-509. http://dx.doi.org/10.1111/1469-0691.12195

[3] Sogaard, M., Norgaard, M., Dethlefsen, C. and Schonheyder, H.C. (2011) Temporal Changes in the Incidence and 30-Day Mortality Associated with Bacteremia in Hospitalized Patients from 1992 through 2006: A Population-Based Cohort Study. Clinical Infectious Diseases, 52, 61-69. http://dx.doi.org/10.1093/cid/ciq069

[4] Mokrzycki, M.H., Zhang, M., Cohen, H., Golestaneh, L., Laut, J.M. and Rosenberg, S.O. (2006) Tunnelled Haemodialysis Catheter Bacteraemia: Risk Factors for Bacteraemia Recurrence, Infectious Complications and Mortality. Nephrology Dialysis Transplantation, 21, 1024-1031. http://dx.doi.org/10.1093/ndt/gfi104

[5] Danish Nephrology Registry (DNR) (2013) Annual Report.

[6] Moore, C.L., Besarab, A., Ajluni, M., et al. (2014) Comparative Effectiveness of Two Catheter Locking Solutions to Reduce Catheter-Related Bloodstream Infection in Hemodialysis Patients. Clinical Journal of the American Society of Nephrology, 9, 1232-1239. http://dx.doi.org/10.2215/CJN.11291113

[7] Marr, K.A., Sexton, D.J., Conlon, P.J., Corey, G.R., Schwab, S.J. and Kirkland, K.B. (1997) Catheter-Related Bacteremia and Outcome of Attempted Catheter Salvage in Patients Undergoing Hemodialysis. Annals of Internal Medicine, 127, 275-280.

http://dx.doi.org/10.7326/0003-4819-127-4-199708150-00003 
[8] Lemaire, X., Morena, M., Leray-Moragues, H., et al. (2009) Analysis of Risk Factors for Catheter-Related Bacteremia in 2000 Permanent Dual Catheters for Hemodialysis. Blood Purification, 28, 21-28. http://dx.doi.org/10.1159/000210034

[9] Saad, T.F. (1999) Bacteremia Associated with Tunneled, Cuffed Hemodialysis Catheters. American Journal of Kidney Diseases, 34, 1114-1124. http://dx.doi.org/10.1016/S0272-6386(99)70018-1

[10] Thomson, P.C., Stirling, C.M., Geddes, C.C., Morris, S.T. and Mactier, R.A. (2007) Vascular Access in Haemodialysis Patients: a Modifiable Risk Factor for Bacteraemia and Death. QJM, 100, 415-422. http://dx.doi.org/10.1093/qjmed/hcm040

[11] Fysaraki, M., Samonis, G., Valachis, A., et al. (2013) Incidence, Clinical, Microbiological Features and Outcome of Bloodstream Infections in Patients Undergoing Hemodialysis. International Journal of Medical Sciences, 10, 1632-1638. http://dx.doi.org/10.7150/ijms.6710

[12] Karkar, A., Bouhaha, B.M. and Dammang, M.L. (2014) Infection Control in Hemodialysis Units: a Quick Access to Essential Elements. Saudi Journal of Kidney Diseases and Transplantation, 25, 496-519. http://dx.doi.org/10.4103/1319-2442.132150

[13] Vanholder, R., Canaud, B., Fluck, R., et al. (2010) Diagnosis, Prevention and Treatment of Haemodialysis Catheter-Related Bloodstream Infections (CRBSI): A Position Statement of European Renal Best Practice (ERBP). Clinical Kidney Journal, 3, 234-246. http://dx.doi.org/10.1093/ndtplus/sfq041

[14] CDC-Centers of Disease Control and Prevention (2016) Core Interventions. http://www.cdc.gov/dialysis/prevention-tools/core-interventions.html

[15] Ethier, J., Mendelssohn, D.C., Elder, S.J., et al. (2008) Vascular Access Use and Outcomes: an International Perspective from the Dialysis Outcomes and Practice Patterns Study. Nephrology Dialysis Transplantation, 23, 3219-3226. http://dx.doi.org/10.1093/ndt/gfn261

[16] Pedersen, C.B. (2011) The Danish Civil Registration System. Scandinavian Journal of Public Health, 39, 22-25. http://dx.doi.org/10.1177/1403494810387965

[17] Friedman, N.D., Kaye, K.S., Stout, J.E., et al. (2002) Health Care-Associated Bloodstream Infections in Adults: a Reason to Change the Accepted Definition of Community-Acquired Infections. Annals of Internal Medicine, 137, 791-797. http://dx.doi.org/10.7326/0003-4819-137-10-200211190-00007

[18] ECDC-European Centre for Disease Prevention and Control (2008) Annual Epidemiological Report on Communicable Diseases in Europe 2008.

http://ecdc.europa.eu/en/publications/Publications/0812_SUR_Annual_Epidemiological_R eport_2008.pdf

[19] Rosenblum, A., Wang, W., Ball, L.K., Latham, C., Maddux, F.W. and Lacson Jr., E. (2014) Hemodialysis Catheter Care Strategies: A Cluster-Randomized Quality Improvement Initiative. American Journal of Kidney Diseases, 63, 259-267. http://dx.doi.org/10.1053/j.ajkd.2013.08.019

[20] Abdul Gafor, A.H., Cheong, P.P., Zainal Abidin, A.F., et al. (2014) Antibiogram for Haemodialysis Catheter-related Bloodstream Infections. International Journal of Nephrology, 2014, 1-6. http://dx.doi.org/10.1155/2014/629459

[21] Alexandraki, I., Sullivan, R., Zaiden, R., et al. (2008) Blood Culture Isolates in Hemodialysis Vascular Catheter-related Bacteremia.The American Journal of the Medical Sciences, 336, 297-302. http://dx.doi.org/10.1097/MAJ.0b013e3181637432

[22] Boel, J., Sogaard, M., Andreasen, V., Jarlov, J.O. and Arpi, M. (2015) Evaluating Antibiotic 
Stewardship Programs in Patients with Bacteremia Using Administrative Data: A Cohort Study. European Journal of Clinical Microbiology \& Infectious Diseases, 34, 1475-1484. http://dx.doi.org/10.1007/s10096-015-2378-x

[23] Mandolfo, S., Acconcia, P., Bucci, R., et al. (2014) Hemodialysis Tunneled Central Venous Catheters: Five-year Outcome Analysis. The Journal of Vascular Access, 15, 461-465. http://dx.doi.org/10.5301/jva.5000236

[24] ECDC-European Centre for Disease Prevention and Control (2013) Antimicrobial Resistance Surveillance in Europe.

http://ecdc.europa.eu/en/publications/Publications/antimicrobial-resistance-surveillance-e urope-2013.pdf

[25] Bakke, C.K. (2010) Clinical and Cost Effectiveness of Guidelines to Prevent Intravascular Catheter-related Infections in Patients on Hemodialysis. Nephrology Nursing Journal, 37, 601-615.

[26] Lindberg, C., Downham, G., Buscell, P., Jones, E., Peterson, P. and Krebs, V. (2013) Embracing Collaboration: a Novel Strategy for Reducing Bloodstream Infections in Outpatient Hemodialysis Centers. American Journal of Infection Control, 41, 513-519. http://dx.doi.org/10.1016/j.ajic.2012.07.015

[27] Lincoln, M. (2011) Preventing Catheter-associated Bloodstream Infections in HemodialysisCenters: the Facility Perspective. Nephrology Nursing Journal, 38, 411-415.

[28] Downham, G., Jones, E., Peterson, P. and Yaser Mourad, M. (2012) Reducing Bloodstream Infections in an Outpatient Hemodialysis Center-New Jersey, 2008-2011. MMWR Morbidity and Mortality Weekly Report, 61, 169-173.

[29] George, A., Tokars, J.I., Clutterbuck, E.J., Bamford, K.B., Pusey, C. and Holmes, A.H. (2006) Reducing Dialysis Associated Bacteraemia, and Recommendations for Surveillance in the United Kingdom: Prospective Study. BMJ, 332, 1435. http://dx.doi.org/10.1136/bmj.332.7555.1435

Submit or recommend next manuscript to SCIRP and we will provide best service for you:

Accepting pre-submission inquiries through Email, Facebook, LinkedIn, Twitter, etc.

A wide selection of journals (inclusive of 9 subjects, more than 200 journals)

Providing 24-hour high-quality service

User-friendly online submission system

Fair and swift peer-review system

Efficient typesetting and proofreading procedure

Display of the result of downloads and visits, as well as the number of cited articles

Maximum dissemination of your research work

Submit your manuscript at: http://papersubmission.scirp.org/

Or contact ojneph@scirp.org 